\title{
Stochastic Acceleration by Turbulence
}

\author{
Vahé Petrosian \\ Physics, Applied Physics, and KIPAC, Stanford University, Stanford, CA 94305
}

\begin{abstract}
The subject of this paper is stochastic acceleration by plasma turbulence, a process akin to the original model proposed by Fermi. We review the relative merits of different acceleration models, in particular the so called first order Fermi acceleration by shocks and second order Fermi by stochastic processes, and point out that plasma waves or turbulence play an important role in all mechanisms of acceleration. Thus, stochastic acceleration by turbulence is active in most situations. We also show that it is the most efficient mechanism of acceleration of relatively cool non relativistic thermal background plasma particles. In addition, it can preferentially accelerate electrons relative to protons as is needed in many astrophysical radiating sources, where usually there are no indications of presence of shocks. We also point out that a hybrid acceleration mechanism consisting of initial acceleration by turbulence of background particles followed by a second stage acceleration by a shock has many attractive features. It is demonstrated that the above scenarios can account for many signatures of the accelerated electrons, protons and other ions, in particular ${ }^{3} \mathrm{He}$ and ${ }^{4} \mathrm{He}$, seen directly as Solar Energetic Particles and through the radiation they produce in solar flares.
\end{abstract}

\section{Introduction}

The presence of energetic particles in the universe has been know for over a century as cosmic rays (CRs) and for a comparable time as the agents producing non-thermal electromagnetic radiation from long wave radio to gamma-rays. In spite of accumulation of considerable data on spectral and other characteristics of these particles, the exact mechanisms of their production remain controversial. Although the possible scenarios of acceleration have been narrowed down, there are many uncertainties about the details of individual mechanisms. Nowadays the agents commonly used for acceleration of particles in astrophysical plasmas can be classified in three categories, namely static electric fields (parallel to magnetic fields), shocks and turbulence. As we will try to show there are several lines of argument indicating that turbulence plays an important role in all these scenarios. In addition, because of the large values of ordinary and magnetic Reynolds numbers, most flows in 
astrophysical plasmas are expected to give rise to turbulence. The generation and evolution (cascade and damping) of plasma turbulence in astrophysical sources is an important aspect of particle acceleration that will be dealt with in other papers of this proceedings. Similarly, electric field and shock accelerations will be discussed by other authors in this proceedings as well. In this paper we discuss particle acceleration by turbulence or plasma waves which is commonly referred to as Stochastic Acceleration (SA for short).

Fermi (1949) was the first author to propose SA as a model for production of CRs, whereby charged particles of velocity $v$, scattering with a rate $D_{\mathrm{sc}}$ in random collisions with moving magnetized clouds of average speed $u$, gain energy at a rate $D_{\mathrm{sc}}(u / v)^{2}$ mainly because energy gaining head on collisions are more numerous than energy losing trailing ones. Nowadays this class of models are often called Second Order Fermi process. Soon after, several authors proposed plasma waves or magnetohydrodynamics (MHD) turbulence as the scattering agents (see e.g. Sturrock 1966; Kulsrud \& Ferarrri 1971, and references therein) 1 In a later paper Fermi (1954) proposed acceleration of particles scattering back and forth between two ends of a contracting magnetic bottle, where the particles gain energy at every scattering so that the acceleration rate is equal to $D_{\mathrm{sc}}(u / v)$, that is linearly with the velocity ratio, hence the name First Order Fermi. For particle velocities $v \gg u$ this is a much faster rate. It is also well known that a particle crossing a convergent flow, e.g. a shock with velocity $u_{\mathrm{sh}}$, gains momentum $\delta p \sim p\left(u_{\mathrm{sh}} / v\right)$. For this reason acceleration by shocks is also referred to as first order Fermi acceleration. However, as described below, this a somewhat of a misnomer because the actual rate of acceleration is proportional to the square of the velocity ratio. Ever since late 1970's when several authors (Krymsky 1977; Axford 1978; Bell 1978; Blandford \& Ostriker 1978) demonstrated that a simple version of this process can reproduce the observed CR spectrum, shock acceleration has been the most commonly invoked process. However, as we will discuss below, in the last couple of decades there has been a renewed interest in SA by turbulence, specially for electrons in radiation producing astrophysical sources from Solar flares to clusters of galaxies.

In the next section we review the relative merits of different acceleration model and in $\S 3$ we give the general formalism used in the SA and other models. In $\S 4$ we will describe application of the SA model to solar flares. A brief summary and conclusions are presented in $\S 5$.

\footnotetext{
${ }^{1}$ For a brief history and more references to other works see Melrose (2009).
} 


\section{Acceleration Models and Turbulence}

We are interested in comparing the various ways of production of energetic particlesstarting from a relatively "cool" background plasma usually having a thermal or Maxwellian distribution with density $n$ and temperature $T 2$ Clearly this must be the first stage of any acceleration process, where the Coulomb collisions, with mean free path $l_{\text {Coul }}=9 \times$ $10^{7} \mathrm{~cm}\left(T / 10^{7} \mathrm{~K}\right)^{2}\left(10^{10} \mathrm{~cm}^{-3} / n\right)$, may be an important, if not the dominant energy loss and particle scattering process. In fact a thermal spectrum requires a high rate for these collisions involving both electrons and protons (and heavier ions) 3 Thus, the first hurdle that any acceleration mechanism must overcome is this loss process. Particles energized at this stage may escape the acceleration region with an energy dependent escape time $T_{\text {esc }}(E)$ favoring escape of the higher energy particles, thus resulting in a population of nonthermal particles, which are observed directly or through the radiation they produce. The escaping particles may be re-accelerated by other mechanisms possibly in collisionless surroundings with size $L<l_{\text {Coul }}$. On the other hand, in a closed system, i.e. when $T_{\text {esc }}$ is larger than the dynamical timescale of the system then, in general, it is difficult to produce a substantial nonthermal tail. As shown in Petrosian \& East (2008) irrespective of the rate or energy dependence of the acceleration process a substantial, if not the bulk, of the energy input goes into heating of the plasma rather than producing a nonthermal electron tail th

The most commonly acceleration mechanisms used for analysis of astrophysical sources are the following:

\section{1. $\quad$ Electric Field Acceleration}

Static electric fields $\mathbf{E}$ parallel to magnetic fields can accelerate a particle with charge $e$ and velocity $v=c \beta$ with the energy gain rate of $\dot{E}=e \mathbf{E} v_{\|}$. If we define the Dreicer field $\mathbf{E}_{\mathrm{D}} \equiv k T /\left(e l_{\text {Coul }}\right) \propto n / T\left(\sim 10^{-5} \mathrm{~V} / \mathrm{cm}\right.$ for solar flare conditions $)$ that results in an energy gain of $\sim k T$ per mean free path, then the energy change over a distance $L$ is given by

$$
\frac{\Delta E}{m_{e} c^{2}}=\frac{\mathbf{E}}{\mathbf{E}_{D}} \frac{m_{e} c^{2}}{k T} \frac{n L}{2.5 \times 10^{23} \mathrm{~cm}^{-2}} .
$$

\footnotetext{
${ }^{2}$ For the purpose of demonstration, in what follows we use numerical values appropriate for solar flares.

${ }^{3}$ From here on, unless specified otherwise we will refer to protons and heavier ions collectively as protons.

${ }^{4}$ We have carried out similar analysis of heating vs acceleration of protons and obtain similar results but on a longer timescale (Kang \& Petrosian, in preparation).
} 
Thus, sub-Dreicer fields can accelerate electrons to relativistic energies only if they extend over large column depths or $N=n L>4 \times 10^{20} \mathrm{~cm}^{-2}\left(T / 10^{7} \mathrm{~K}\right)$. Since the rate of energy gain per unit length is independent of particle mass (because as defined $\mathbf{E}_{D} \propto m_{e}^{2} c^{4}$ ), for acceleration of proton to relativistic regime $\left(E \sim m_{p} c^{2}\right)$ we need a column depth of $\sim$ $10^{24} \mathrm{~cm}^{-2}\left(T / 10^{7} \mathrm{~K}\right)$. For example, in solar flare coronal loops, with $T \sim 10^{7} \mathrm{~K}$ and column depth $\sim 10^{20} \mathrm{~cm}^{-2}$, particles can be accelerated up to only 10 's of $\mathrm{keV}$, far below the required 10 's of MeV electrons or $>\mathrm{GeV}$ protons. Column depths and temperatures in astrophysical sources (e.g. $N \sim 10^{22} \mathrm{~cm}^{-2}$ and $T \sim 10^{8} \mathrm{~K}$ for intra cluster medium; $N \sim 10^{21} \mathrm{~cm}^{-2}$ and $T \sim 10^{4} \mathrm{~K}$ in typical galactic HII regions, etc) are also not sufficient for acceleration of electrons or protons to relativistic energies. Super-Dreicer fields can accelerate particles to higher energies but since now the acceleration rate is higher than Coulomb energy loss rate this can lead to runaway particles and an unstable bump-in-tail distribution which will give rise to turbulence. In addition, it is difficult to sustain large scale electric fields in a highly conducting ionized plasma unless the resistivity is anomalously high (Tsuneta 1985; Holman 1985). After the pioneering work by Speiser (1970) it was assumed that the electric fields induced by reconnection are the agents of acceleration (see also Litvinenko 1996, 2003; La Rosa et al. 2006) but recent particle-in-cell (PIC) and MHD simulations of reconnection (Drake et al. 2006; see also Cassak et al. 2006; Zenitani \& Hoshino 2005) present a more complicated picture and show that turbulence may be an important ingredient. Thus, electric fields cannot be the sole agent of acceleration, but they may produce turbulence, which can accelerate particles and possibly enhance the reconnection rate, as suggested by Lazarian \& Vishniac (1999).

\subsection{Fermi Acceleration}

As mentioned above there are two types of Fermi acceleration, first order in a shock and second order in a turbulent plasma. As also indicated above the former has been invoked often in astrophysical situations primarily because it is believed to be a faster mechanism of acceleration and the environment surrounding a supernova shock seems well suited for production of CR protons. However, there are many shortcomings in the original elegant models developed in late 1970's for this mechanism. Since then a great deal of work has gone into the the development of this model and in addressing its shortcomings. These include injection of seed particles, losses (specially for electrons), and escape and nonlinear effects (see e.g. Drury 1983; Blandford \& Eichler 1987; Jones \& Ellison 1991; Malkov \& Drury 2001; Diamond \& Markov 2007; Beresnyak et al. 2009). More importantly, a shock by itself cannot accelerate particles and requires scattering agents that can cause the repeated passages

across the shock front, especially for a parallel shock with magnetic field parallel to flow 
velocity. The most likely agent is turbulence and the rate of acceleration is governed again by the scattering rate by turbulence, and the acceleration rate, $\dot{E} / E \sim \dot{p} / p \propto D_{\mathrm{sc}}\left(u_{\mathrm{sh}} / v\right)^{2}$ (see below), is no longer first order in the velocity ratio. Although there are indications that magnetic field and turbulence may be generated by the upstream accelerated particles (see e.g. Bell 1978), many details of the microphysics remain unsolved. Two stream (or another plasma) instability is a possible mechanism for generation of turbulence but there are indication that this may be suppressed in a turbulent medium (Yan \& Lazarian 2002). Exact determination of the scattering rate requires knowledge of the intensity and spectrum of the turbulence which determine $D_{\mathrm{sc}}$ but are essentially unknown. Usually Bohm diffusion is assumed 5

Second order Fermi or SA process, on the other hand, occurs always at some level because turbulence in addition to scattering can also accelerate particles directly. As is well known, relativistic particles in weakly magnetized plasma (e.g. that in a supernova shock) are scattered at a faster rate than the rate of acceleration by turbulence, so that acceleration by a shock is deemed to be faster. This, however, is not always the case, specially for acceleration of electrons in radiating sources. As we will discuss in more detail below, Pryadko \& Petrosian (1997; PP97) showed that at low energies and/or in strongly magnetized plasmas the acceleration or energy diffusion rate by turbulence exceeds the scattering rate and therefore exceeds the acceleration rate by a shock. Thus, under these circumstances the main objection of slowness of SA does not apply. For example, in the case of solar flares Hamilton \& Petrosian (1992) show that SA by a modest level of whistler waves can accelerate the background particles to high energies within the desired time (see also Miller and Reames 1996).

We can conclude then that irrespective of which process of acceleration is at work, turbulence always has a major role. Moreover, as we will see in the next section, in practice, i.e. mathematically, there is little difference between first and second order Fermi acceleration (see e.g. Jones 1994).

There are, of course, other acceleration processes similar to those discussed above on the microphysics level but phenomenologically different. One such process is that proposed by Drake et al. (2006) occurring via the interactions of particles with the "islands" produced in their PIC simulations. Another is the process proposed by Fisk \& Glockler (2010) for acceleration in the solar wind. These will be discussed in other sections of these proceedings.

\footnotetext{
${ }^{5}$ First order Fermi acceleration may also occur in the converging flow in the reconnection region (de Gouvia del Pinto \& Lazarian 2005; see also Lazarian's contribution here.
} 


\section{BASIC EQUATIONS}

Interactions of particles with turbulence, which is a common ingredient of all acceleration processes, are dominated by many weak rather than few strong scattering events. In this case the Fokker-Planck formalism provides the best description of the particle kinetics which can have different forms depending on circumstances. The basic equations here are well known and have been described in many papers in the past. In this section we briefly review these equations and point out two important features of plasma wave-particle interactions not broadly known or acknowledged. These features have important effects on the relative importance of first and second order Fermi acceleration, and on the relative acceleration rates of electrons vs protons, ${ }^{3} \mathrm{He}$ vs ${ }^{4} \mathrm{He}$ and other ions.

\subsection{Particle Kinetic Equations}

Most astrophysical plasmas are strongly magnetized so that the gyro-radius of particles (with mass $m$ ),$r_{g}=1.7 \times 10^{3} \mathrm{~cm} \beta \gamma\left(\mathrm{G} / B_{\perp}\right)\left(\mathrm{m} / \mathrm{m}_{e}\right)$, is much smaller than the scale of the spatial variation of the field. In this case particles are tied to the magnetic field lines and instead of dealing with temporal evolution of the distribution of energetic particles in six dimensions (3 space, 3 momentum) one deals with the gyro-phase averaged distribution which depends only on three variables; spatial coordinate $s$ along the field lines, the momentum $p$, the pitch angle or its cosine $\mu$, and of course also time 6 Then, the evolution of the particle distribution, $f(t, s, p, \mu)$, can be described by the Fokker-Planck equation as particles undergo stochastic scattering and acceleration by interaction with plasma turbulence (with diffusion coefficients $D_{p p}, D_{\mu \mu}$ and $D_{p \mu}=D_{\mu}$ ) and suffer losses (with rate $\dot{p}_{L}$ ) due to other interactions with the plasma particles and fields. The particles may also gain energy in presence of shocks or large scale electric fields (with the rate $\dot{p}_{G}$ ):

$$
\frac{\partial f}{\partial t}+v \mu \frac{\partial f}{\partial s}=\frac{1}{p^{2}} \frac{\partial}{\partial p} p^{2}\left[D_{p p} \frac{\partial f}{\partial p}+D_{p \mu} \frac{\partial f}{\partial \mu}\right]+\frac{\partial}{\partial \mu}\left[D_{\mu \mu} \frac{\partial f}{\partial \mu}+D_{\mu p} \frac{\partial f}{\partial p}\right]-\frac{1}{p^{2}} \frac{\partial}{\partial p}\left(p^{2} \dot{p} f\right)+\dot{S}
$$

Here $\dot{p}=\dot{p}_{G}-\dot{p}_{L}$ is the net momentum change rate and $\dot{S}$ is a source term, which could be the background thermal plasma or some injected spectrum of particles. The effect of the magnetic field convergence or divergence can be accounted for by adding $\frac{c \beta d \ln B}{d s} \frac{\partial}{\partial \mu}\left(\frac{\left(1-\mu^{2}\right)}{2} f\right)$ to the right hand side. And if there are large scale flows with velocity $u$ and spatial gradient $\frac{\partial u}{\partial s}$ along the field lines, e.g. around a shock front, their effects can be accounted for by

\footnotetext{
${ }^{6}$ Here we use $p$ and $\mu$, or particle energy (in $m c^{2}$ units) $E=\gamma-1$ and $\mu$, instead of $p_{\|}$and $p_{\perp}$.
} 
adding the term

$$
\frac{1}{3} \frac{\partial u}{\partial s} \frac{1}{p^{2}} \frac{\partial}{\partial p}\left(p^{3} f\right)-\frac{\partial u}{\partial s} f
$$

to the right hand side. In general, this complete equation is rarely used to determine the particle distribution $f(t, s, p, \mu)$. Instead the following approximations are used to make it more tractable.

Pitch-angle isotropy: If the pitch angle diffusion rate is high so that the scattering time $\tau_{\text {sc }} \sim 1 / D_{\mu \mu}$ is shorter than all other time scales $\left(\tau_{\text {diff }} \sim p^{2} / D_{p p}, \tau_{\text {cross }}=L / v, \tau_{\mathrm{L}}=\right.$ $p / \dot{p}_{L}, \tau_{\mathrm{ac}}=p / \dot{p}_{G}$, etc, where $L$ is the size of the interaction region), then the pitch angle distribution of the particles will be nearly isotropic. If we define

$$
F(t, s, p) \equiv \frac{1}{2} \int_{-1}^{1} d \mu f(t, s, p, \mu), \quad \dot{Q}(t, s, p) \equiv \frac{1}{2} \int_{-1}^{1} d \mu \dot{S}(t, s, p, \mu),
$$

then the kinetic equation simplifies to the Diffusion-Convection Equation (see, e.g. Kirk et al. 1988; Dung \& Petrosian 1994)

$$
\frac{\partial F}{\partial t}=\frac{\partial}{\partial s} \kappa_{s s} \frac{\partial F}{\partial s}+\frac{1}{p^{2}} \frac{\partial}{\partial p}\left(p^{4} \kappa_{p p} \frac{\partial F}{\partial p}-p^{2}\langle\dot{p}\rangle F\right)+p \frac{\partial \kappa_{s p}}{\partial s} \frac{\partial F}{\partial p}-\left(\frac{1}{p^{2}} \frac{\partial F}{\partial s} \frac{\partial}{\partial p}\left(p^{3} \kappa_{s p}\right)\right)+\dot{Q}(s, t, p),
$$

where $\langle\ldots\rangle$ implies pitch angle averaged values and the three transport coefficients are related to the diffusion coefficients as

$$
\begin{aligned}
\kappa_{s s} & =\left(v^{2} / 8\right) \int_{-1}^{1} d \mu\left(1-\mu^{2}\right)^{2} / D_{\mu \mu}, \\
\kappa_{s p} & =v /(4 p) \int_{-1}^{1} d \mu\left(1-\mu^{2}\right) D_{\mu p} / D_{\mu \mu}, \\
\kappa_{p p} & =1 /\left(2 p^{2}\right) \int_{-1}^{1} d \mu\left(D_{p p}-D_{\mu p}^{2} / D_{\mu \mu}\right) .
\end{aligned}
$$

This approximation is generally valid for high energy particles interacting with Alfven waves in a plasma with relatively low magnetization, i.e. Alfven velocity $v_{A}=\sqrt{\left(B^{2} /(4 \pi \rho)\right.} \ll v$, where $B$ is the magnetic field strength and $\rho$ is the gas density 7 so that

$$
R_{1} \equiv D_{p p} /\left(p^{2} D_{\mu \mu}\right)=\tau_{\text {sc }} / \tau_{\text {diff }} \sim\left(v_{A} / v\right)^{2} \ll 1
$$

However, as mentioned above, PP97 have shown that, in certain situations (low energies and high magnetic fields), the energy diffusion rate $\sim D_{p p} / p^{2}$ may exceed the pitch angel

\footnotetext{
${ }^{7}$ Note that this is the common definition of Alfven velocity which could exceed speed of light. However the actual phase velocity of Alfven waves is equal to $v_{A} / \sqrt{1+v_{A}^{2} / c^{2}}<c$.
} 
diffusion rate $\sim D_{\mu \mu}$ so that $R_{1} \gg 1$, invalidating the above approximation. In this case, the momentum diffusion is the dominant term and the kinetic equation (2) again simplifies to

$$
\frac{\partial f^{\mu}}{\partial t}+v \mu \frac{\partial f^{\mu}}{\partial s}=\frac{1}{p^{2}} \frac{\partial}{\partial p}\left(p^{2} D_{p p}^{\mu} \frac{\partial f^{\mu}}{\partial p}-p^{2} \dot{p} f^{\mu}\right)+\dot{S}^{\mu}
$$

where now one must include the $\mu$ dependence of all terms and of the distribution function. In general, there will be significant acceleration only if the energy diffusion and acceleration times are shorter than the crossing time $\tau_{\text {cross }}$. In addition, if the scattering time $\tau_{\text {sc }}(\mu) \sim$ $1 / D_{\mu \mu}$, though now longer than momentum diffusion time, is also shorter than $\tau_{\text {cross }}=l / v$, and/or if the other coefficients (most importantly those related to acceleration) vary slowly with $\mu$, then the particle distribution will again be nearly isotropic 8 and we can use pitch angle averaged distribution $F(t, s, p)$ and coefficients

$$
\left\langle D_{p p}\right\rangle=\frac{1}{2} \int_{-1}^{1} d \mu D_{p p}(\mu)
$$

and (similarly) $\left\langle D_{\mu \mu}\right\rangle,\left\langle\dot{p}_{G}\right\rangle$ and $\left\langle\dot{p}_{L}\right\rangle$. As described in the caption of Figure 1 (right) use of these averaged quantities is justified. In addition, now the term $v \mu \partial f^{\mu} / \partial s$ should be replaced by the spatial diffusion term $\frac{\partial}{\partial s} \kappa_{s s} \frac{\partial F}{\partial s}$. Thus, we can ignore $\mu$ dependences, in which case the two equations are almost identical: They have different spatial dependence terms (e.g. terms involving $\partial \kappa_{s p} / \partial s ; \partial F / \partial s$ ), but more importantly, have two different momentum diffusion forms; one for the case $R_{1}<1$ in Equation (8) and the second for $R_{1}>1$ given by Equation (11).

Spatial Homogeneity: A second simplification, which applies to both $R_{1}<1$ and $R_{1}>1$ cases, can be used if the acceleration region is homogeneous (i.e. $\partial / \partial s=0$ ), or if one deals with a spatially unresolved source where one is interested in spatially integrated equations. In this case it is convenient to define $N(t, E) d E=\int d V\left[4 \pi p^{2} F(t, s, p) d p\right]$ and replace the spatial diffusion term (or the advection term in Equation [10]) plus other terms involving $\partial F / \partial s$ by an escape term with an escape time $T_{\text {esc }}(E)$ defined by

$$
\int d V 4 \pi p^{2} \frac{\partial}{\partial s}\left(\kappa_{s s} \frac{\partial F}{\partial s}-F \frac{1}{p^{2}} \frac{\partial}{\partial p}\left(p^{3} \kappa_{s p}\right)\right)=\frac{N(t, E)}{T_{\mathrm{esc}}(E)} .
$$

Then we obtain the well-known equation

$$
\frac{\partial N}{\partial t}=\frac{\partial}{\partial E}\left(D_{E E} \frac{\partial}{\partial E} N\right)-\frac{\partial}{\partial E}\left(\left[A(E)-\dot{E}_{L}\right] N\right)-\frac{N}{T_{\mathrm{esc}}}+\dot{\mathcal{S}}, \text { with } T_{\mathrm{esc}}=\tau_{\mathrm{cross}}+\frac{\tau_{\text {cross }}{ }^{2}}{\tau_{\mathrm{sc}}}
$$

\footnotetext{
${ }^{8}$ Note also that Coulomb scatterings with pitch angle diffusion rate $D_{\mu \mu}^{\text {Coul }} \propto n /\left(\beta^{3} \gamma^{2}\right)$ can also contribute to the scattering rate, specially at low energies, and help to isotropize the pitch angle distribution.
} 
where $\dot{E}_{L}=p \dot{p}_{L} / \gamma$ is the energy loss rate. This clearly is an approximation with the primary assumption being that the transport coefficients vary slowly spatially, e.g. $\partial \kappa_{s p} / \partial s \ll$ $\left.\left\langle\kappa_{s p}\right\rangle / L\right), \int 4 \pi p^{2} d p \kappa_{i} F d V \sim\left\langle\kappa_{i}\right\rangle N d E$, etc. Note that here $\dot{\mathcal{S}}(t, E)$ and $N(E, t) / T_{\text {esc }}$ represent the rates of injection and escape of particles in and out of the acceleration site, and that $T_{\text {esc }}$ as defined can account for the spatial diffusion term in equation (5) when $\tau_{\text {cross }} / \tau_{\text {sc }} \gg 1$ and the advection term in equation (10) when of $\tau_{\text {cross }} / \tau_{\text {sc }} \ll 1$.

This equation is fairly general and can handle different acceleration scenarios. For example for the SA by turbulence the energy diffusion and the direct acceleration coefficients are related as 9

$$
D_{E E}=v^{2} \bar{D}_{p p} \text { and } A(E)=\left(D_{E E} / E\right)\left[\left(2 \gamma^{2}-1\right) /\left(\gamma^{2}+\gamma\right)\right]
$$

where $\bar{D}_{p p}$ is equal to $p^{2} \kappa_{p p}$ for the isotropic case (Equation 5) and is equal to $\left\langle D_{p p}\right\rangle$ for equation (10). As stressed above turbulence is present in all acceleration scenarios so these energy diffusion and the direct acceleration rates are the minimum rates. However if there are other diffusion or acceleration mechanisms we should add their contribution. For example, if the acceleration volume contains a converging flow (as in a shock) with velocity $u$, then there will be additional direct acceleration rate $A_{u}(E)=p\langle\partial u / \partial s\rangle$. Or, at low energies and for a high density plasma, as mentioned above, one should add the effects of pitch angle and energy diffusion due to Coulomb collisions.

Finally, for completeness we mention that for most astrophysical situations the primary contribution to the loss rate for energetic electrons comes from Coulomb collisions at low energies (which as stated at the outset are essential in establishing a thermal distribution) and synchrotron and inverse Compton at high energies, and for protons from elastic Coulomb collisions and inelastic strong interactions with background protons and other ions. In certain cases there may be catastrophic losses through which particles are taken out of the system.

In summary then, it turns out that this most commonly used transport equation in astrophysical problems is a good approximation at all energies and all degrees of magnetization for spatially unresolved sources.

\footnotetext{
${ }^{9}$ Sometimes the energy diffusion term on the right hand side of equation (13) is written as $\frac{\partial^{2}}{\partial E^{2}}\left(D_{E E} N\right)$ in which case we need to add $d D_{E E} / d E$ to the right hand side of the direct acceleration term $A(E)$.

${ }^{10}$ In this case the term $-u F$ should be added inside the large parenthesis in Equation (12).
} 


\subsection{Two Important Features}

There are two noteworthy aspects to the formalism described above.

1. The first feature is related to the fact that the relative rates of the momentum and pitch angle diffusion, and hence the ratio $R_{1}$, vary with plasma conditions and particle energy. This has two important consequences.

The first is that both rates increase with decreasing energy and/or increasing level of turbulence (see Equation [17] below). As a result SA of low energy thermal particles in magnetized plasma, the type usually encountered in astrophysical radiating sources, is not slow as the second order name would imply. In deed, in recent years this has been recognized and SA has found application in many sources involving acceleration of low energy background electrons.

The second has to do with the change of the ratio $R_{1}$. In Figure 1 (left, from PP97) we show contour maps of this ratio in the electron energy and degree of magnetization space represented by the parameter $\alpha=\omega_{p} / \Omega \propto \sqrt{n} / B$, the ratio of plasma to gyro frequency. On the middle panel we show variation of $R_{1}$ (from Petrosian \& Liu, 2004, PL04) with energy for several values of $\mu$ for both electrons and protons interacting with parallel propagating plasma waves. These figures show the regions of the phase space where $R_{1}>1$ and where the SA by turbulence is the dominant process. Let us consider a simple non relativistic hydrodynamic shock or a parallel shock with magnetic field parallel to the shock flow velocity 11 The SA rate is $\sim \bar{D}_{p p} / p^{2}$ while the shock acceleration rate is proportional to fractional energy gain per crossing $\delta p / p \sim u_{\mathrm{sh}} / v$ divided by average crossing time $\delta t \sim \kappa_{s s} / v u_{\mathrm{sh}} \sim\left(v / u_{\mathrm{sh}}\right) D_{\mu \mu}^{-1}$ (Krymsky et al. 1979; Lagage \& Cesarsky 1983; Drury 1983) so that the shock acceleration rate $A_{\mathrm{sh}} \sim D_{\mu \mu}\left(u_{\mathrm{sh}} / v\right)^{2}$ is no longer a first order mechanism, and the ratio $A_{\mathrm{SA}} / A_{\mathrm{sh}} \sim R_{1}\left(v / u_{\mathrm{sh}}\right)^{2}>R_{1}$. This means that when $R_{1}>1$, which is the case at low energies, the SA is the more dominant of the two mechanisms. Thus, the following picture seems to emerge. When a particle crosses the shock to the downstream turbulent region its interactions with plasma waves increase its energy substantially before it has a chance to cross the shock. Only when its energy has increased to a sufficiently large value to make the ratio $R_{1}<1$, then it can undergo repeated passage across the shock 12 Only

\footnotetext{
${ }^{11}$ For perpendicular shock one gets similar result with added effect of the ratio $\kappa_{\|} / \kappa_{\perp}$ of the diffusion parallel and perpendicular to the field lines (see e.g. Giacalone 2005a \& 2005b and references therein).

${ }^{12}$ This, of course requires turbulence in the upstream region as well whose origin is not well understood. Possible generation by some instability due to accelerated particles has been suggested and some details have been worked out by Lee (2005), but this problem is not fully resolved as observations of shocks in the solar wind do not always show presence of any turbulence in the upstream region.
} 
when its energy has increased to a sufficiently large value to make a $R_{1}<1$, then it can undergo repeated passage across the shock, hence beginning a second stage of acceleration by the shock. Note also that even at high energies and interactions with low frequency fast modes with phase velocity equal to Alfven velocity when $R_{1} \sim\left(v_{A} / v\right)^{2}$ is less than one, the ratio of the SA to shock acceleration rates

$$
A_{\mathrm{SA}} / A_{\mathrm{sh}} \sim\left(v_{A} / u_{\mathrm{sh}}\right)^{2} \sim 1 /\left(\beta_{p} \mathcal{M}^{2}\right),
$$

which could be greater than one for low beta plasmas $\left(\beta_{p}=2\left(u_{\text {Sound }} / v_{A}\right)^{2}<1\right)$ and for shocks with low Mach number $\mathcal{M}=u_{\text {sh }} / u_{\text {Sound }}$. Thus, we can think of the acceleration as a hybrid mechanism with turbulence providing the initial rise in energy of background plasma till they become energetic enough to be accelerated also by the shock during which SA continues and may not be negligible. This sort of behavior can be seen in the recent PIC simulation by Sironi \& Spitkovsky (2009) where test particles appear to gain energy gradually in the downstream region till they cross the shock and get a jump in energy (see their Figure 8). This hybrid scenario, in a way, also solves the long standing "injection problem" in shock acceleration requiring injection of high energy (i.e pre accelerated) particles, especially for perpendicular shocks.
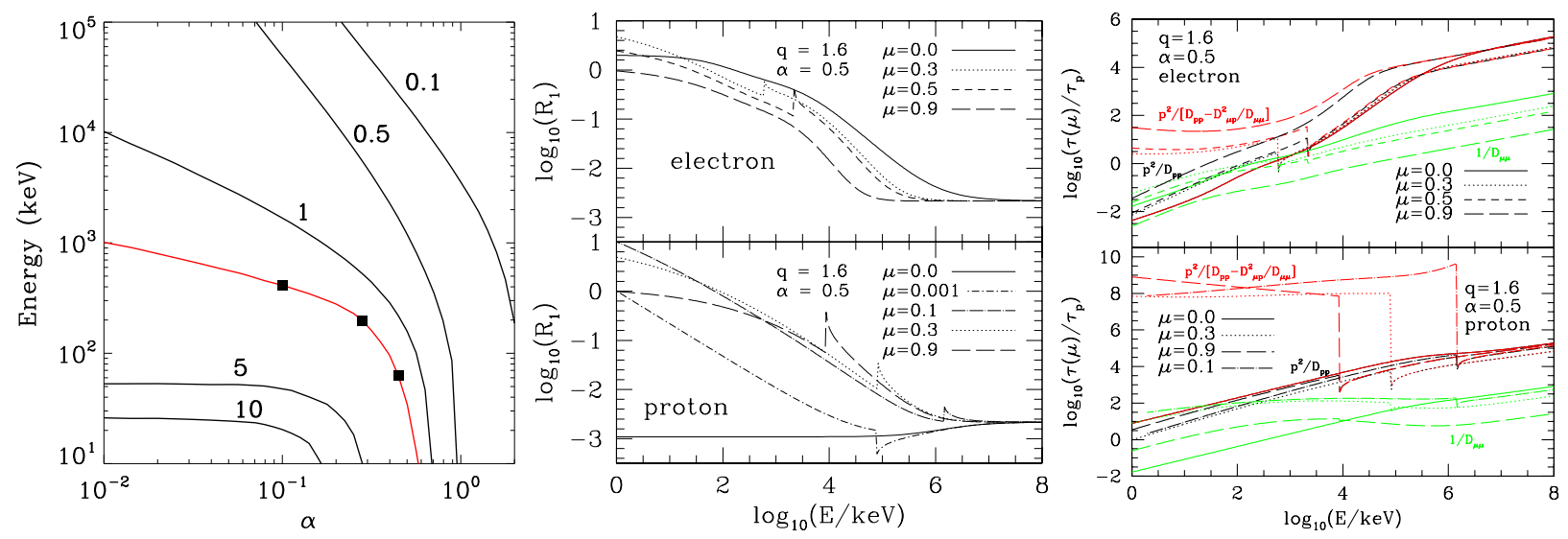

Fig. 1. Left: Contours of the ratio $R_{1}$ in the electron energy and parameter $\left.\alpha=2.3(100 \mathrm{G} / B) \sqrt{(} n / 10^{10} \mathrm{~cm}^{-3}\right)$ for $\mu=0$. The red line shows the $R_{1}=1$ contour for $\mu=0.3$. Regions below $R_{1}=1$ curves are when acceleration by SA becomes dominant (from PP97). Middle: Variation with energy of the ratio $R_{1}$ for different pitch angles of electrons and protons Note that the acceleration rate can exceed scattering up to $\sim 100 \mathrm{keV}$ for most pitch angles (From PL04). Right: Variations of timescales associated with rates of pitch angle diffusion (green), the simple momentum diffusion (black), and the momentum diffusion for the isotropic case with coefficient $\kappa_{p p}$ (red). The latter is considerably longer than the others at energy ranges when resonance with one mode is dominant. This effect is much more pronounced for protons than electrons. Note also that the shapes of $D_{\mu \mu}$ and $D_{p p} / p^{2}$ associated curves are similar for different pitch angles making use of pitch angled averaged quantities a reasonable approximation.

2. The second interesting feature has to do with the difference between the energy diffusion and acceleration rates in the two limiting cases (Equations 5 and 10) derived above. For a resonant interaction with plasma waves in general the three Fokker-Planck diffusion 
coefficients obey the following simple relations (see e.g. PL04 for parallel and Pryadko \& Petrosian 1999, for perpendicular propagating waves):

$$
\left(D_{p p} / p^{2}\right):\left(D_{\mu p} / p\right): D_{\mu \mu}=\left[x_{j}^{2}\right]:\left[x_{j}\left(1-\mu x_{j}\right)\right]:\left[\left(1-\mu x_{j}\right)^{2}\right] \quad \text { with } \quad x_{j}=\left(v_{p h, j} / v\right)^{2}
$$

where $v_{p h}=\omega / k$ is the phase velocity of the plasma mode (with frequency $\omega$ and wave vector $k$ ) in resonance with the particle of momentum $p$ and pitch angle cosine $\mu$. This implies that for interaction with a single wave the acceleration rate as given in Equation (8), would be zero 13 However, in general interactions with many waves contribute to each coefficient so that this rate is never zero, but if the interaction with one mode is dominant then the rate becomes much smaller than normal diffusion rate $\left\langle D_{p p} / p^{2}\right\rangle$. Figure 1 (right) shows the energy dependence at various values of $\mu$ of the acceleration (and scattering) times based on the two forms of the acceleration rate (black vs red curves). As evident there is considerable difference between the two rates specially at low energies, and the differences are more pronounced for protons compared to electrons. As discussed below, this is important for the relative SA rates of different species in particular for electrons vs protons. We will also show that a similar process affects the relative acceleration of ${ }^{3} \mathrm{He}$ and ${ }^{4} \mathrm{He}$ in solar flares.

\section{Stochastic Acceleration in Solar Flares}

Over the past several decades there has been considerable discussion of first vs second order acceleration (see e.g. Drury 1983) and the role of turbulence in the latter (see e.g. Hall \& Sturrock 1967; Kulsrud \& Ferarrri 1971). However the success of shock acceleration in producing the observed $\mathrm{CR}$ spectrum has relegated SA by turbulence to be a less important process even though the importance of the role played by turbulence in scattering of the energetic particles is fully appreciated. As shown above, there are many similarities between the two processes, and in some cases SA by turbulence may be the dominant process. This fact has been recognized in more recent times and there has been renewed activity in application of the SA model to several astrophysical sources. Most prominent among these is the Solar Flare which is the most developed and will be discussed in more detail below. But SA has been applied to nonthermal emission from accretion disks around black holes: e.g. Sgr A* black hole in the center of the milky way (Liu et al, 2004, 2006a and 2006b), other active galactic nuclei (Stawartz \& Petrosian 2008), stellar size black holes (Li \& Miller 1997), gamma-ray bursts (Lazarian et al. 2003), to supernovae shocks (Scott \& Chevalier 1975; Cowsik \& Sarkar 1984; Fan et al. 2009; Virtanen \& Vainio 2005), to giants radio

\footnotetext{
${ }^{13}$ This can be ascertained by plugging in for the diffusion coefficients in Equation (8) the expressions in Equation (16).
} 
galaxy lobes (Lacombe 1977; Achterberg 1979; Eilek 1979), and to intra cluster medium of clusters of galaxies (Petrosian 2001, Brunetti \& Lazarian 2007).

\subsection{Basic Scenario for Solar Flares}

The complete development of a solar flare involves many phases. After a complex preflare build up of magnetic fields, the first phase is the reconnection and the process of the energy release. The final consequences of this released energy are the observed radiations from radio to gamma-rays, Solar Energetic Particles (SEPs), and Coronal Mass Ejections (CMEs). The basic scenario for these processes, as depicted by the cartoon in Figure 2 (left), can be summarized as follows: Even though it is generally agreed that the flare energy comes from the annihilation of magnetic fields via reconnection, the exact mechanisms of the release and dissipation of this energy remains controversial. Dissipation can occur via Plasma Heating, Particle Acceleration or Plasma Turbulence. As stated above turbulence is a necessary ingredient for acceleration and, as we will outline below, there is considerable observational evidence favoring SA by turbulence. Thus we believe that most of the magnetic energy is converted into turbulence near or above the top of a coronal loop, which we refer to as the acceleration site or the loop top (LT) region. The turbulence undergoes nonlinear wave-wave interactions causing a dissipationless cascade to smaller scales. The wave-particle interaction results in damping of the turbulence, heating of the plasma and acceleration of particles. The accelerated particles are somewhat trapped at the LT because of their short mean free path due to scattering by turbulence which enhances their radiation intensity there. Eventually these particles escape the turbulent LT region. Some escape along open field lines and may undergo further scattering and acceleration by a CME shock during their transport to the Earth where they are detected as SEPs 14 Most of the particles travel down the legs of the loop and produce the observed flare radiation; microwaves via synchrotron, hard X-rays (HXR) via bremsstrahlung produced by electrons, and gamma-rays via nuclear line excitations and decay of pions (primarily $\pi^{0}$ ) produced by protons, along the loop, but primarily at its foot points (FPs). However most of the energy of the accelerated particles is dissipated in the chromosphere and below via inelastic Coulomb collisions. This deposited energy causes heating and evaporation of the plasma up into coronal loops, which then produces the bulk of the flare radiation in the forms of thermal soft X-rays and optical photons. The evaporation changes the the density and temperature in the corona which can affect the reconnection, energy release and acceleration processes.

\footnotetext{
${ }^{14}$ The escaping electrons may also produce type-III and other radio radiation.
} 


\subsection{Some Relevant Observations of Flares}

Observations of solar flares are reviewed by Raymond et al. and some theoretical aspects, namely those on global aspects of energy release and acceleration are reviewed by Cargill et al. in this proceedings. Here we discuss the confrontation between the accelerations models and observations. A successful model must account for all observations. However, some observation are more critical than others. Here we focus on the following three separate observed characteristics, explanation of which we believe constitutes as minimum requirement for models.

1. Radiative signatures of electrons, primarily from o̊bservations.

2. Relative acceleration of electrons and protons.

3. Isotopic abundance enhancements in SEPs, especially that of ${ }^{3} \mathrm{He}$, and the variation of abundance ratio and spectra of ${ }^{3} \mathrm{He}$ and ${ }^{4} \mathrm{He}$.

We have made extensive comparisons of the SA model with these observations and find numerous signatures of electrons and protons that support the model. Below we present a brief description of the above observations and how the SA model can account for them.

\subsubsection{Radiative Signatures of Electrons}

Radiation by accelerated electrons produce wealth of observation, and we clearly cannot address them all here. Instead we focus on a few critical observations, mainly from RHESSI, that are related to the acceleration process.

- One observation which we believe provides the most compelling and direct evidence for the presence of turbulence in the LT (acceleration) site is the observation by Yohkoh (Masuda et al. 1994) showing a distinct impulsive HXR emission from the LT in addition to the usual FP sources. This apparently is present in almost all Yohkoh flares (Petrosian et al. 2002) and analysis of flares has confirmed this picture (Liu et al. 2003; Krucker \& Lin 2008) for essentially all limb flares. The fact that we see LT and FP emission but little or none from the legs of the loop requires lingering of electrons in the LT region for times longer than the crossing time $\tau_{\text {cross }}=L / v$. This requires an enhanced scattering near the LT region. Petrosian \& Donaghy (1999) show that Coulomb scattering cannot be the agent for this trapping because then the electrons will also lose most of their energy in the LT region on the same timescale and never reach the FPs. The most likely scattering agent is turbulence.

This turbulence can also accelerate the electrons. There may be other acceleration at work too, but as described above, at low energies and for solar flare conditions (low $\beta$ plasma) SA 
by turbulence is the most effective process.

- In rare cases, when the FP sources are weak or occulted, one can see a double LT source (Figure 2 middle, from Liu et al. 2008; see also Sui \& Holman, 2003) as expected in the model depicted on the left. This simple picture also predicts a gradual rise of the LT source accompanied with a continuous increase in separation of the FPs as the reconnection proceeds and larger closed loops are formed, a feature that has been seen at other wavelength but it is usually difficult to see in HXRs in weak flares because of low signal to noise ratio and in most strong flares because they tend to have complicated loop structures. The Nov. 3, 2003 $\mathrm{X}$-class limb flare consisting of a single loop provided a good opportunity to see this feature in HXRs. The right panel of Figure 2 (from Liu et al. 2004) clearly shows this behavior.
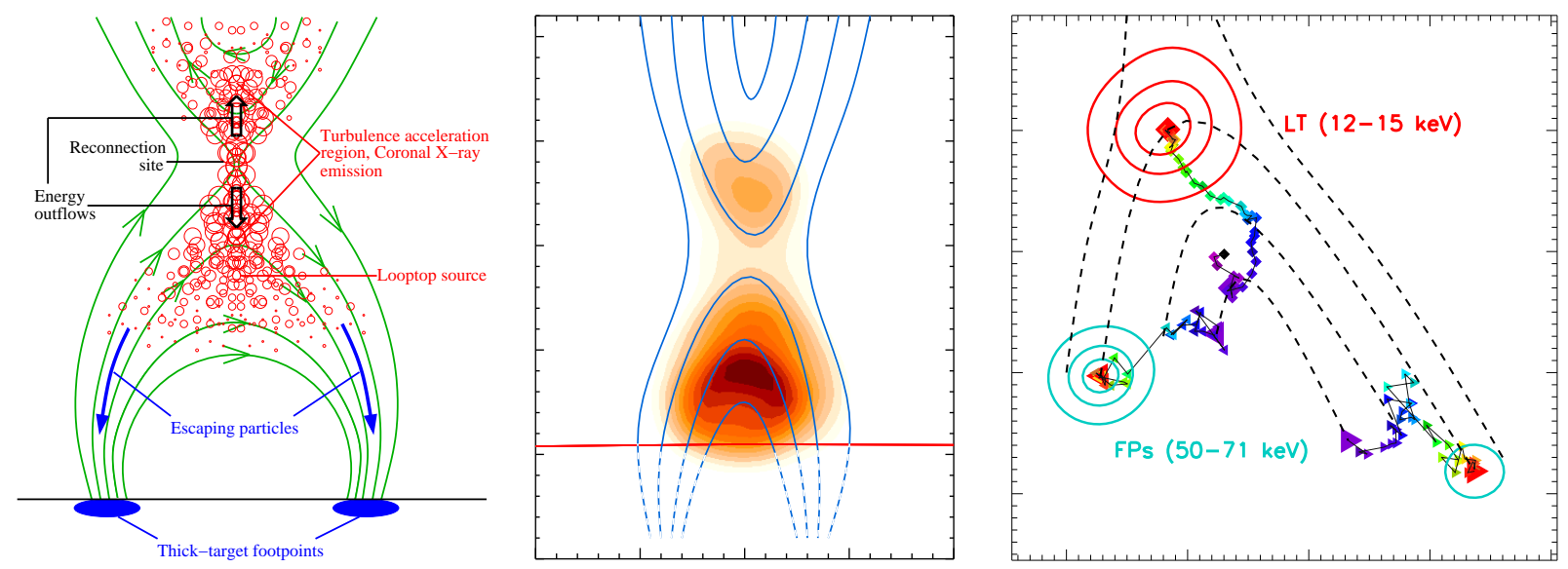

Fig. 2. - Left: A schematic representation of the reconnecting field forming closed loops and coronal open field lines. The red foam represents PWT. Middle: Image of the flare of April 30, 2002, with occulted FPs showing two distinct coronal sources as expected from the model in the left. The curves representing the magnetic lines (added by hand) show the occulted FPs below the limb (red line) (from Liu et al. 2008). Right: Temporal evolution of LT and FP HXR sources of the Nov. 3, 2003 flare. The symbols indicate the source centroids and the colors show the time with a 20 sec interval, starting from black (09:46:20 UT) and ending at red (10:01:00 UT) with contours for the last time. The curves connecting schematically the FPs and the LT sources for different times show the expected evolution for the model at the left (from Liu et al. 2004).

- Another important observation by is the relative spectra of LT and FP sources. The LT source is often dominated by a very hot thermal type emission with a relatively soft tail, while the FP sources consist of harder power laws with little or no thermal part as shown by the example in Figure 3 (left). These are exactly the kind of spectra that come out from models of SA by turbulence shown in the right panel of Figure 3.15 Figure 3 (left) also shows a forward fit of the observed spectra to those obtained from SA model with the specified acceleration parameters.

\footnotetext{
${ }^{15}$ It should, however, be noted that most generic acceleration models accelerating particles from a hot plasma and with scattering provided by turbulence will produce a hot quasi-thermal plus a nonthermal tail (Petrosian \& East 2008).
} 
- Flares during the impulsive phase often show a soft-hard-soft temporal evolution and sometimes a slower than expected (assuming only losses) temperature decline in the thermal decay phase (McTiernan et al. 1993). The results in Figure 3 (right) agree with these evolutionary aspects as well. As can be seen the spectra electrons accelerated by turbulence get harder with increasing value of the wave-particle interaction rate parameter

$$
\tau_{p}^{-1}=(\pi / 2) \Omega f_{\text {turb }}(q-1)\left(c k_{\min } / \Omega_{e}\right)^{q-1}
$$

where $f_{\text {turb }}=(\delta B / B)^{2}$ is the ratio of the turbulence to magnetic field energy density with wave energy spectral index of $q$ for wave vectors $k>k_{\min }$. Thus, as the level of turbulence or $f_{\text {turb }}$ increases and decreases during the impulsive phase, we go from a thermal to softhard-soft nonthermal and back to a thermal phase.

- In several RHESSI limb flares Jiang et al. (2006) find that during the decay phase the LT source continues to be confined (not extend to the FPs), and that the observed energy decay rate is much lower than the Spitzer (1962) conduction rate. These observations require suppression of the conduction and a continuous input of energy during the decay phase. A low level of lingering turbulence can be the agent for both.
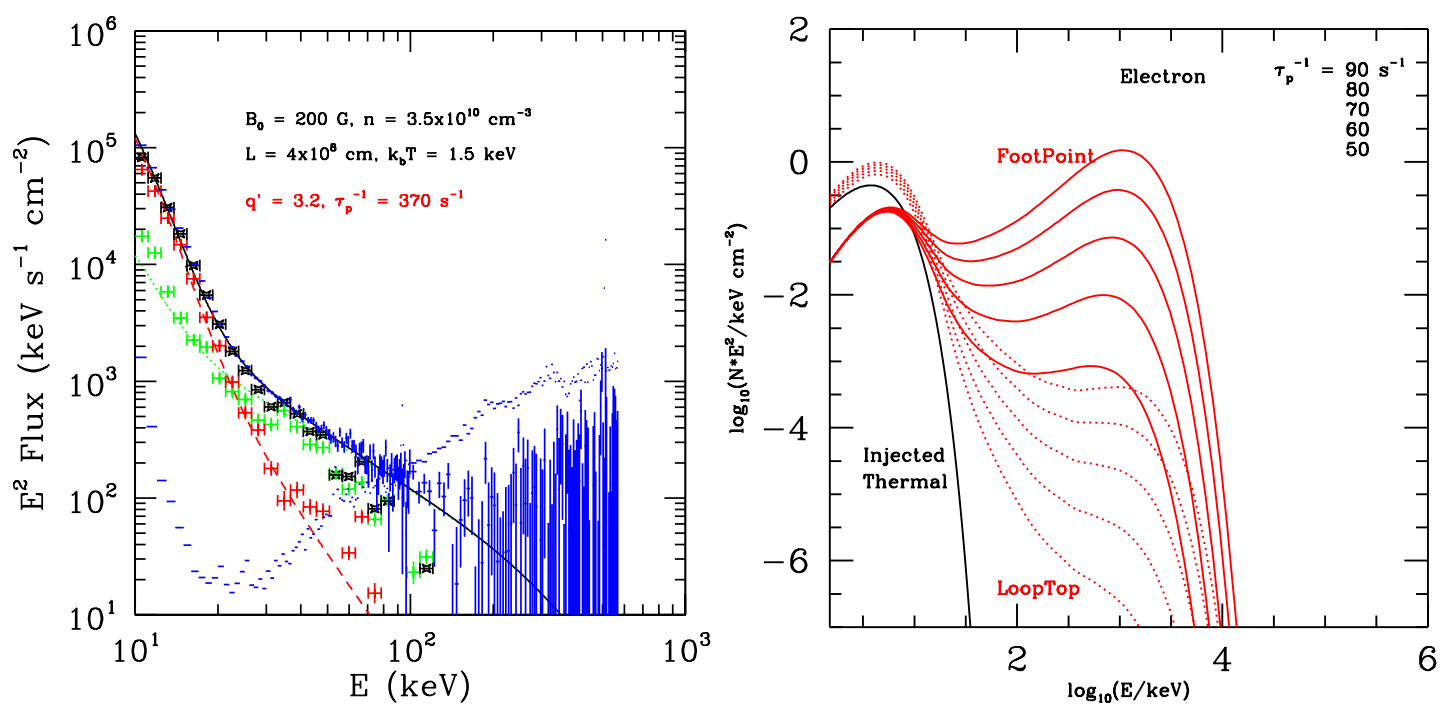

Fig. 3.- Left: A fit to the peak time total (black), FPs (green) and LT (red) spectra of a 9/20/2002 flare observed by RHESSI. The dashed and dotted lines are bremsstrahlung spectra by electrons whose spectrum is calculated using Equations (13) with the indicated acceleration parameters, showing the presence of a quasi-thermal (LT) and a nonthermal component. The solid line gives the sum of the two. The blue dashes indicate the level of the background radiation (Liu et al. 2003). Right: The dependence on $\tau_{p}^{-1} \propto f_{\text {turb }}$ of the accelerated electron spectrum $E^{2} N(E)$ at the LT (dotted) and the effective thick target spectrum $\left(E^{2} / \dot{E}_{L}\right) \int_{E}^{\infty} d E^{\prime} N\left(E^{\prime}\right) / T_{\text {esc }}\left(E^{\prime}\right)$ at the FPs (solid). Higher levels of turbulence produce harder spectra and more acceleration than heating (from PL04). 


\subsubsection{Relative Acceleration Rates of Electrons and Protons}

Flares are generally recognized based on radiative and other (heating and evaporation) signatures of the accelerated electrons. As one would expect protons will also be subjected to same acceleration mechanisms. The radiative signatures of protons are (i) narrow gamma-ray lines in the 1-7 MeV range arising from de-excitation of nuclei of ions excited by accelerated protons (or viceversa, which produce broader lines), and (ii) $>70 \mathrm{MeV}$ continuum emission from decay of pions produced in $p-p$ interaction (mainly from decay of $\pi^{0}$ 's). Gamma-rays are generally observed in large and gradual flares, but this is partly due to relatively lower sensitivity of past gamma-ray detectors compared to HXR detectors. However, Fermi with its superior sensitivity is beginning to observe gamma-rays from modest M-class flares (see Ackermann et al. 2012). The SA model has been the working hypothesis for acceleration of protons as well. (See pioneering works by Ramaty (1979) and collaborators; e.g. Murphy et al. 1987).

There have been considerable discussions of relative energies of populations of accelerated electrons and protons but most recent analysis of HXR and gamma-ray emission of a large sample of flares by Shih et al. (2009) show a good correlation but with relatively broad dispersion (see Figures 3 of Raymond et al. in this proceedings. As this figure shows the mean value of the ratio of energy in accelerated electrons (obtained from HXR fluxes) to that of protons (deduced from gamma-ray fluxes) is larger than 1 while this ratio in galactic CRs presumably accelerated in supernova shocks is much smaller than one. In Figure 4 (left) we show the same distribution from the above mentioned figure (in blue) along with the distribution of the same ratio we obtained from observations of SEP electrons and protons (for the same energy ranges, taken from data compiled by Cliver \& Ling 2007, in red). We will return to the differences between the two histograms below. But for now we focus on the fact that the acceleration mechanism operating in flares seems to put more energy in

electrons over protons compared with the mechanism responsible for acceleration of SEPs and galactic CRs. In shock acceleration models (with Alfvenic turbulence as the source of scattering) one would expect a more efficient acceleration of protons compared to electrons. This may indicate that a different mechanisms is at work in solar flares. As shown below this is another evidence that SA (rather than a shock) is the dominant acceleration mechanism in flares.

In PL04 we address this problem by looking at the details of the SA by parallel propagating waves of a thermal population of electrons and protons. We find in general that for typical flare conditions this mechanism favors acceleration of electrons than protons. This is because electrons and protons undergo resonant interactions with different plasma modes of different wave vectors or frequencies. In general protons have fewer resonances than electrons 

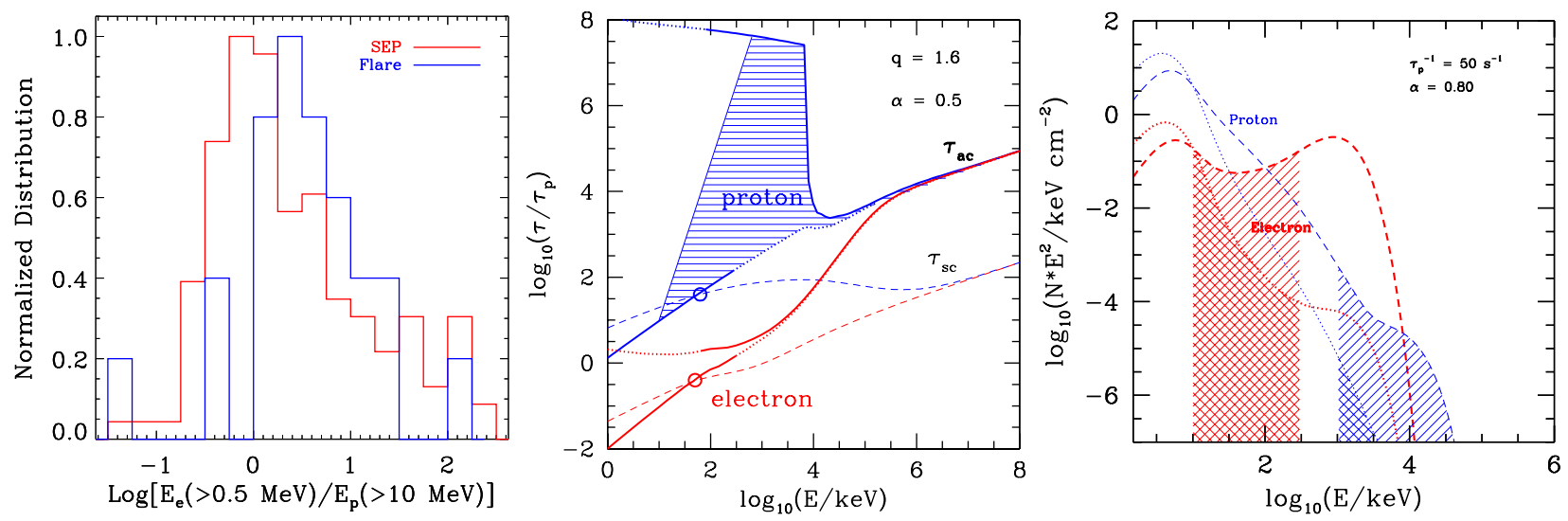

Fig. 4. - Left: Normalized distribution of the electron to proton energy flux ratios for flares derived from the data in Shih et al, (2009, blue) and SEPs from data in Cliver \& Ling (2007, red) showing preference for acceleration of electrons in flares. Middle: Scattering (dashed) and acceleration (solid; given by Equation 8) time scales in unit of $\tau_{p}$ for protons (blue) and electrons (red) showing a large barrier or long acceleration time for protons in the 0.1 to $10 \mathrm{MeV}$ range (dashed region) due to the dominance of a single resonance mode. This barrier disappears at low energies once $\tau_{\mathrm{sc}}$ becomes longer than momentum diffusion time $p^{2} / D_{p p}$, and at high energies where there is more than one dominant resonant mode (from PL04). Right: Spectra of accelerated electrons (red) and protons (blue) at the acceleration site (dotted) and the effective thick target FP spectra $\left(E^{2} / \dot{E}_{L}\right) \int_{E}^{\infty} d E^{\prime} N\left(E^{\prime}\right) / T_{\text {esc }}\left(E^{\prime}\right)$ (dashed) for given values of $\tau_{p}$ and $\alpha \propto \sqrt{n} / B$ and for temperature $k T=1.5 \mathrm{keV}$ which produces more nonthermal electrons compared to protons in their respective observed ranges shown by the dashed areas (from PL04).

so that it is more likely that they will have one dominant resonant mode. In this case, as described in the second of Two Important Features in $§ 3.2$, and shown in Figure1(right), the rate of acceleration given by equation (8) becomes very small hindering the acceleration of protons. Figure 4 (middle) shows presence of a large barrier against acceleration of proton, manifested by a large acceleration timescale, resulting in acceleration of fewer protons in the energy range from few $\mathrm{MeV}$ to $\mathrm{GeV}$ range needed for production of gamma-rays (lines and continuum). As mentioned above, the acceleration rate by a shock also depends on the rate of wave-particle interactions. However, in this case it depends on the spatial diffusion coefficient $\kappa_{s s}$ which in turn depends only on $D_{\mu \mu}$ and not the combination of the diffusion coefficient in equation (8) which affects the SA rate. The right panel of Figure 4 shows the accelerated electron and proton spectra (multiplied by square of energy) and the effective thick-target spectra for the specified values of the basic acceleration parameters (namely density, magnetic field, temperature and level and spectrum of turbulence represented by the parameter $\tau_{p}$ ) which produce more nonthermal electrons than protons in their respective observes ranges shown by the hashed areas.

However, as shown in PL04, the ratio of electron to proton acceleration varies considerably with the basic acceleration parameters such as $\tau_{p}, \alpha \propto \sqrt{n} / B$ and temperature of the injected particles. In fact, as can be seen from comparison of spectra shown in the left and middle pane of Figure 5 with that in Figure 4 left a small change in $\alpha$ can produce a 
large change in the ratio of the energies of the accelerated electrons to protons explaining the broad distributions seen in the left panel of Figure 4. Similarly, as evident fro spectra shown in the right panel of Figure 5 higher temperature of background particles also favor the acceleration of protons. One consequence of these is that the proton acceleration will be more efficient in larger (and most likely lower $B$ value) loops and at late phases, when evaporation increases the temperature and density $n$ (hence the value of parameter $\alpha$ ) 16 This can explain the difference in centroids of HXR and gamma-ray emission seen by ås described by Raymond et al. in these proceedings. It should be emphasized, however, that here we have concentrated on the ratios of accelerated electrons and protons in the energy ranges that produce the observed HXRs and gamma-rays (electrons $>10$ 's of keV, protons $>10 \mathrm{MeV}$ ). But as can be seen from above figures, in general, there are considerable number of accelerated protons at lower energies which do not produce detectable radiation. We will discuss the role of these particles below.

In summary, the SA model can account for various differences seen in acceleration of electrons and protons.
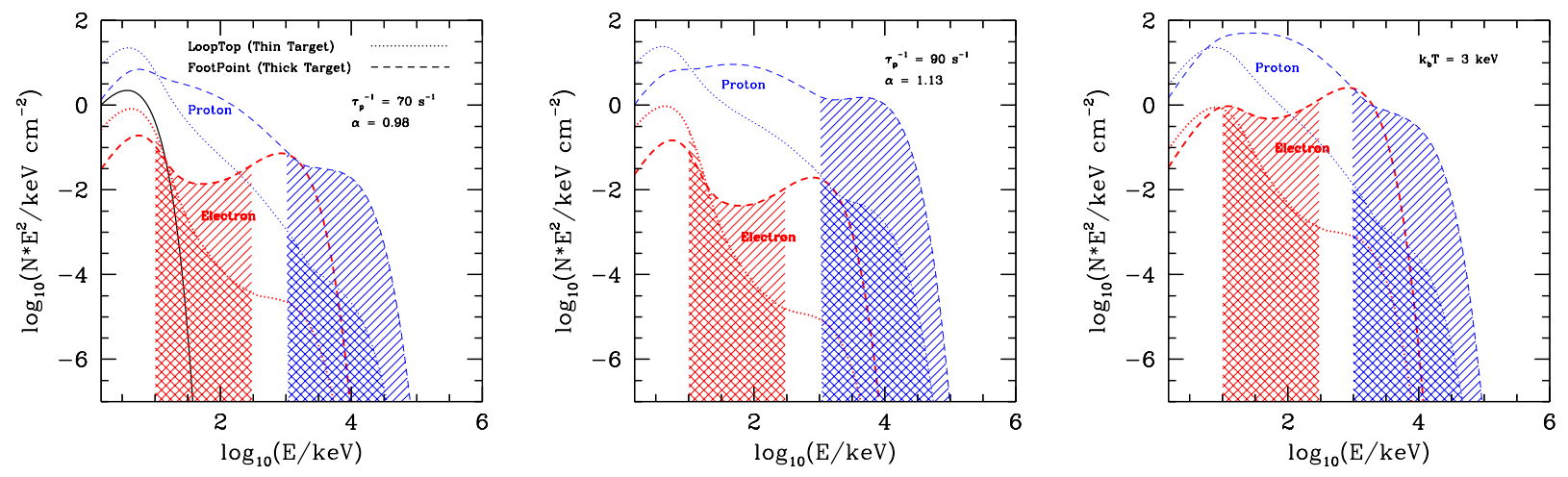

Fig. 5. - Same as the left panel of Figure 4 but for different values of $\tau_{p}, \alpha \propto \sqrt{n} / B$ and $k T$ showing large variations in the relative accelerations of electrons vs protons. Left: $\tau_{p}^{-1}=70 \mathrm{~s}^{-1}, \alpha=0.98, k T=1.5 \mathrm{keV}$. Middle: $\tau_{p}^{-1}=90 \mathrm{~s}^{-1}, \alpha=1.13$, $k T=1.5 \mathrm{keV}$. Right: $\tau_{p}^{-1}=70 \mathrm{~s}^{-1}, \alpha=0.98, k T=3.0 \mathrm{keV}$. As evident higher densities, higher temperatures and lower magnetic fields favor acceleration of protons vs electrons and viceversa. Note also that the quasi-thermal proton component of less than one $\mathrm{MeV}$, which do not produce gamma-rays, can escape and possibly re-accelerated by a CME shock, and be observed as SEPs. This can explain the shift to the right of the SEP (red) distribution shown in the left panel of Figure 4 (From PL04.)

\footnotetext{
${ }^{16}$ Note that a similar difference was also predicted by Miller \& Roberts (1995) based on other grounds.
} 


\subsubsection{SEP Spectra and Abundances}

It is commonly believed that the observed relative abundances of ions in SEPs favor the SA model (e.g. Mason et al. 1986; Mazur et al. 1995). More recent observations and modelings have confirmed this picture (see Mason et al. 2000, 2002; Reames et al. 1994, 1997; Ng \& Reames 1994; Miller 2002).

One of the most vexing problem of SEPs has been the enhancement of ${ }^{3} \mathrm{He}$. Observations show a wide range of ${ }^{3} \mathrm{He}$ to ${ }^{4} \mathrm{He}$ ratios; ranging from photospheric values in gradual-strong flares to values several thousand times larger in impulsive-weak flares. It should be emphasized that there are not two distinct classes (impulsive and gradual) with a well defined bimodal distribution. Rather, as indicated by observations (Ho et al. 2005), there is a broad continuum of events as shown in the left panel of Figure 6, going from weak, short duration (impulsive) events with strong enrichments at one end to long (gradual), strong and normal abundances events at the other extreme end. It was recognized early that the unusual charge to mass ratio of ${ }^{3} \mathrm{He}$ could be the cause here. However, these early works did not provide a satisfactory quantitative explanation. 17

With a more complete treatment of ${ }^{3} \mathrm{He}$ and ${ }^{4} \mathrm{He}$ acceleration, Liu, Petrosian \& Mason 2004 and 2006 (LPM04, LPM06) have demonstrated that SA can indeed explain the extreme enhancement of ${ }^{3} \mathrm{He}$ and can also reproduce the observed ${ }^{3} \mathrm{He}$ and ${ }^{4} \mathrm{He}$ spectra for high enrichment cases. The reason for success of our approach is in a way similar to our treatment of election versus proton acceleration, where inclusion of resonance interactions with multiple wave modes gives rise to the different rates. In case of ${ }^{3} \mathrm{He}$ and ${ }^{4} \mathrm{He}$ we also find that, once the effects of ionized He ( $\alpha$ particles) are included in the description of the dispersion relation, the low energy ${ }^{3} \mathrm{He}$ ions have more resonances than ${ }^{4} \mathrm{He}$ ions, as a result of which they are more readily accelerated than ${ }^{4}$ He. In LPM04 and LPM06 it was shown that with this model we can obtain an excellent fit to the observed spectra of several weak shorter duration events for both ${ }^{4} \mathrm{He}$ and ${ }^{3} \mathrm{He}$ (which show the characteristic convex spectral shapes) for reasonable plasma and acceleration parameters. An example of an excellent fit (with $\left.\tau_{p}^{-1}=190, \alpha=0.5\right)$ is shown in the right panel of Figure 6 with the solid lines. On this plot we also show two other sets of spectra for two different values of the SA rate parameter $\tau_{p}^{-1}$, which is proportional to the level of turbulence (see Equation 17) and perhaps to the overall strength of the flare. As can be seen in all cases most of the ${ }^{3} \mathrm{He}$ are accelerated into the observed range, owing to the high efficiency of their acceleration, while this is true for ${ }^{4} \mathrm{He}$ only for large values of $\tau_{p}^{-1}$, i.e. for high levels of turbulence. For small levels there is a barrier for ${ }^{4} \mathrm{He}$, similar to that for protons in Figure 4, so that most of the ${ }^{4} \mathrm{He}$ appear

\footnotetext{
${ }^{17}$ For a brief review of these earlier works see Petrosian (2008).
} 
as a low energy bump (a quasi-thermal component), with only a small fraction reaching the observed range. However, the number of ${ }^{4} \mathrm{He}$ ions in this range increases rapidly with increasing value of $\tau_{p}^{-1}$. This also explains the wide range and the trend of the fluence ratios. As shown in the right panel of this figure the model predicted ratio decreases with increasing levels of turbulence and hence increasing ${ }^{4} \mathrm{He}$ fluence. This trend is independent of the other SA model parameters (like the parameter $\alpha$ as can be seen in this figure). It also turns out that this model can reproduce the observed distributions of fluences of both ions (see Petrosian et al. 2009).
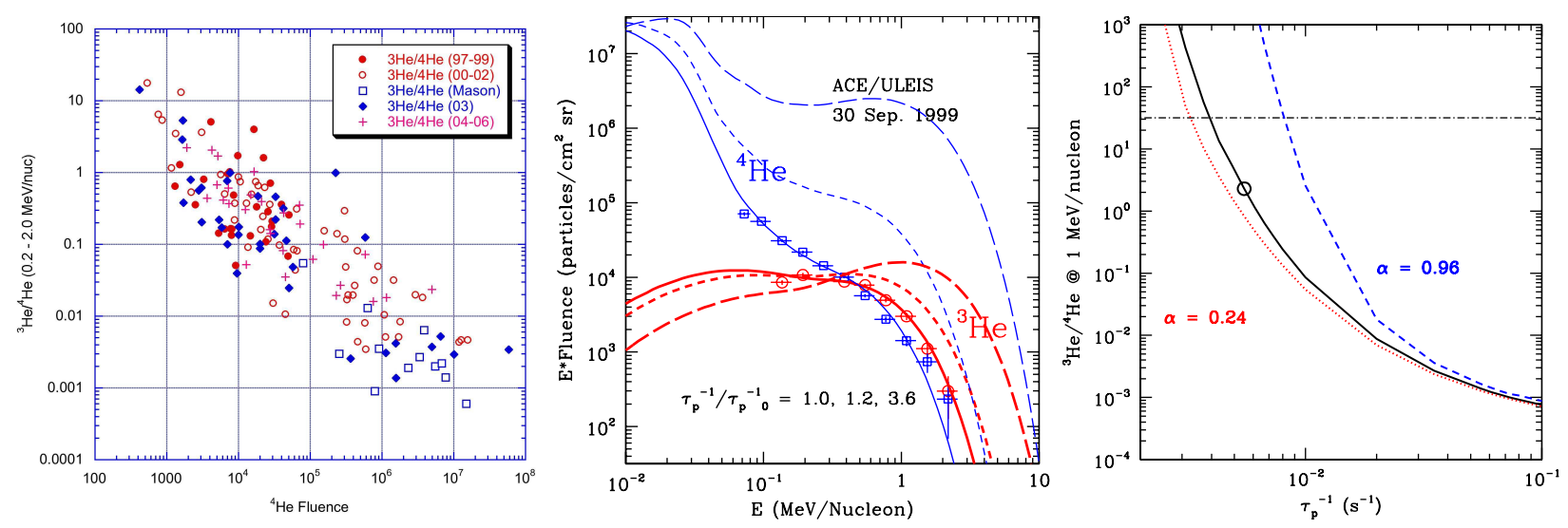

Fig. 6. - Left: Observed variation of Ratio of fluences of ${ }^{3} \mathrm{He}$ and ${ }^{4} \mathrm{He}$ vs the fluence of ${ }^{4} \mathrm{He}$ showing a wide range of ${ }^{3} \mathrm{He}$ enhancement decreasing with increasing event fluence. This also shows a broader distribution of ${ }^{4} \mathrm{He}$ than ${ }^{3} \mathrm{He}$ fluences (from Ho et al. 2005). Middle: Model fit to ${ }^{3} \mathrm{He}$ and ${ }^{4} \mathrm{He}$ spectra of Sep. 30, 1999 event observed by $A C E$ showing an excellent fit (solid lines) for $\alpha=1$ and the specified values of the rate parameter $\tau_{p}^{-1}$ or the level of turbulence $\left(\tau_{p 0}=0.0055\right)$. Also shown are two sets of model spectra with different levels of turbulence (or $\tau_{p}^{-1}$ ). Note that in all three cases almost all of the ${ }^{3} \mathrm{He}$ are accelerated into a nonthermal component while at lower levels of turbulence most of ${ }^{4} \mathrm{He}$ form a low energy bump in the unobserved range with a smaller high energy tail. But with increasing level of turbulence more ${ }^{4}$ He ions are accelerated into the observed range (from Petrosian et al. 2009). Right: Model calculation of the variation of the accelerated ${ }^{3} \mathrm{He}$ to ${ }^{4} \mathrm{He}$ fluence ratio (at $E=1 \mathrm{MeV} /$ nucleon) with level of turbulence or $\tau_{p}^{-1}$ for three values of $\alpha \propto \sqrt{n} / B$ (note that $\alpha=1$ for the black line). Note also that the trend and the range of the ratio mimics the observation shown in the left panel (from LPM06).

It should, however, be emphasized that even though we obtain the observed low ratio of fluences for stronger events, the spectra of ${ }^{4} \mathrm{He}$ obtained for these events (e.g. the long dashed line in Figure 6, right) do not agree with the observations (we discuss a possible remedy for this in the next section). Nevertheless, this is a significant breakthrough in understanding of SEPs. There is also an increasing enhancement with increasing mass of the ion. Possible explanations of these and other aspects of the enrichments can be found in a recent review by Petrosian (2008; e.g. Figure10). Clearly more work is required for a complete description of all observed characteristics of SEPs, some of which may require another mechanism of acceleration as discussed next 


\subsection{The Role of CME Shocks}

We have shown that the SA model can reproduce many of the observed features but there are several aspect that need refinements or introduction other processes. For example, we have seen that even though this model can explain the predominance of the accelerated electrons at the flare site and the broad range of the accelerated electron to proton flux ratios it cannot account for the difference between the relative rates of accelerations of electrons and protons as deduced by the radiations they produced at the flare site and that observed in SEPs which favor proton acceleration. We have also indicated we can account for the varied observed spectra and the broad range of the isotopic enrichments in particular that of the ${ }^{3} \mathrm{He}$ in weaker, more impulsive flares, while the ${ }^{4} \mathrm{He}$ model spectra for high fluence-long duration events seems quite different than that observed in such events. The model spectra are softer and unlike the observed (broken) power laws. Thus, the spectra of the accelerated particles coming out of the flare site must be modified by a secondary process to agree with observations.

As is well known, gradual strong flares are associated with CMEs which has led to the idea that the shock produced by the CME can be responsible for acceleration of SEPs. However, as it usually the case with shock acceleration, the question of seed particles is uncertain here as well. It is unlikely that the cold background particles in high corona are the seeds. Tylka \& Lee (2006) in their phenomenological study of acceleration by a shock were able to produce the observed spectra of the SEP assuming a "suprathermal" seed population, extending to higher energies for perpendicular shocks. It then seems natural to assume that the seed particles are flare accelerated ions which are then re-accelerated by the shock. The ${ }^{4}$ He spectra of high fluence events shown in Figure 6 (right) have this kind of characteristics making them good candidates for re-acceleration.

This leads us to the following scenario. The flare site acceleration is the first and primary stage of acceleration and is common to all events. A second phase acceleration can occur in the CME shocks which could modify the spectrum of SEPs escaping the flare site. The possibility that there may be two acceleration mechanisms at work is not a new idea. The new aspect of this scenario is that we have a hybrid acceleration model, where the seeds of the second stage (re)acceleration by the CME shock are the flare site particles accelerated by

turbulence 18 As shown above, the spectrum of particles escaping the flare site consists of two components: a low energy quasi-thermal component (which is below the observable energy range of 0.1 to $10 \mathrm{MeV} /$ nucleon) and a higher energy nonthermal component with high ${ }^{3} \mathrm{He}$

\footnotetext{
${ }^{18}$ In fact there is evidence that energetic ( $>0.1 \mathrm{MeV} /$ nucleon) ions are "present upstream of all interplanetary shocks" (Desai et al. 2003).
} 
enrichment. This scenario has the attractive feature that even though the nonthermal tails may be highly enriched (as in impulsive flares), the total (quasi-thermal plus nonthermal) number of seed particles injected into the CME shock can have essentially normal abundances and harder (power-law) spectra. Thus, for events near the gradual end both components are re-accelerated leading to a near normal abundances.

In Figure 7 we compare the SEP observations of the gradual 18 Jan. 2000 event with small isotopic enhancement (points) with the re-accelerated spectra (solid lines) obtained using the flare accelerated spectra (dashed lines) as the source term $\dot{Q}(E)$ in Equation (13) that are re-acceleration, with an addition of direct acceleration term $A_{\mathrm{sh}}(E)=A_{0} E^{2}$, presumably due to a CME shock, with of $A_{\mathrm{sh}} \sim A_{S A}$ at $0.1 \mathrm{MeV} /$ nucleon. The resultant spectra are harder and closer to normal abundance ratio. We hasten to add that these are preliminary explorations and the agreement, though not perfect, is very good considering that we have used a simple power law acceleration rate. A better agreement can be achieved with a more realistic form for the acceleration rate.

A similar scenario can also explain the differences between the distributions of electron to proton energy flux ratio deduced from observations at the flare site and from SEPs shown in Figure 4 (left). As discussed in $§ 4.2 .2$, in general SA at the flare site is more efficient in acceleration of electrons than protons that have energies capable of producing the observed gamma-rays. However, as also emphasized in $§ 4.2 .2$, just as is the case for ${ }^{4} \mathrm{He}$, flare accelerated protons have a substantial low energy component which can be re-accelerated at a CME shock to yield a smaller electron to proton ratio in SEPs as compared to flares.

\subsection{Testing the Model}

In a new and possibly far reaching work (Petrosian \& Chen 2010), we have initiated determination of some of the important parameters of the kinetic equation (13) (left) directly from the observed data instead of the usual forward fitting method, like that shown in Figure 3, where one assumes values and energy dependences for these parameters, calculates the particle distribution and its radiative spectrum, and then fits to the data (see e.g. Park et al. 1997). In our new work, using the recently developed regularized inversion technique by Piana et al. (2003 and 2007), we are able to determine the energy dependence of the escape and scattering times due to turbulence as shown in Figure 7 (right). An important aspect of this result is that the escape time increases with energy (and as a result the scattering time decreases) relatively rapidly in disagreement with the expected behavior for SA by parallel propagating plasma waves with a Kolmogorov type spectrum (see PP97 or PL04). The observed behavior requires a steeper than Kolmogorov spectrum so that we are dealing 
with wave vector values in the steep damping range. This discrepancy could also be an indication that the simple SA model used in above mentioned papers requires modification. For example, inclusion of the effects of the convergence of the magnetic fields in the LT region, as can be seen in Figure 2 (left), can produce an escape time similar to the timescale for Coulomb collisions which increases with energy. Or our simplified description of the escape time as defined in Equation (13) may require modification. Another possibility is that the outflows from the reconnection region may produce standing shocks that can modify the acceleration rate relative to the scattering rate. These are interesting possibilities that eie intend be explored in future works.
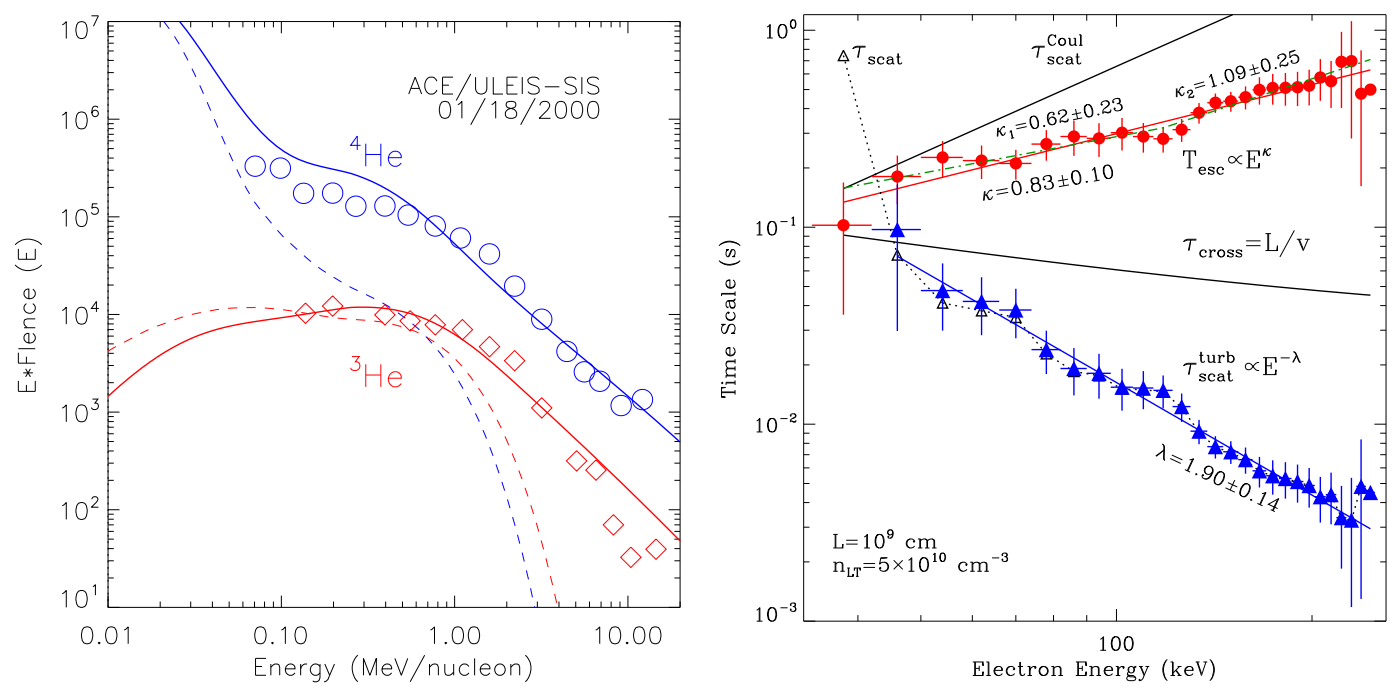

Fig. 7.- Left: Comparison with observed ${ }^{3} \mathrm{He}$ and ${ }^{4} \mathrm{He}$ spectra of event on 18 Jan 2000 with our proposed model, where the flare site accelerated spectra (dashed lines) for an intermediate value of $\tau_{p}^{-1}$ (see Figure 6) are used for re acceleration by a combined shock and SA processes. Middle: Variation with energy of the escape and scattering times due to turbulence obtained directly from RHESSI data using inversion technique for the Nov. 3, 2003 flare. In contrast to the model calculated $T_{\text {esc }}$ in PL04, the observed time scale increases with energy (from Petrosian \& Chen 2010).

\section{Summary and Conclusion}

1. We have reviewed merits of different acceleration mechanisms and pointed out that in general turbulence plays a significant role in all of them so that SA of particles by the turbulence, the original mechanism proposed by Fermi, is omni-present.

2. We have shown that at low energies and for highly magnetized plasmas SA by turbulence is the most efficient mechanism and that it can accelerate particles as rapidly as required in most astrophysical radiation sources. Thus, SA is an attractive scenario for acceleration of background thermal particles. 
3. We have also pointed out that in the presence of shocks a hybrid process of initial SA acceleration by turbulence and subsequent re-acceleration by the shock is what may be operating in many situations providing an answer to the long standing question of origin of the seed particles for shock acceleration.

4. We have shown that a closer analysis of wave-particle interactions in a turbulent plasma also leads to interesting differences between the acceleration rates of electrons and protons and among heavier ions, in particular ${ }^{3} \mathrm{He}$ and ${ }^{4} \mathrm{He}$. Low density, high magnetic fields preferentially accelerate electrons while the opposite is true for protons. In case of ${ }^{3} \mathrm{He}$ and ${ }^{4} \mathrm{He}$ we find that ${ }^{3} \mathrm{He}$, by virtue of its resonance with more modes than ${ }^{4} \mathrm{He}$ is accelerated readily in most circumstances, while efficient acceleration of ${ }^{4} \mathrm{He}$ to high energies requires high levels of turbulence.

5. We have demonstrated these aspects of acceleration mechanism using solar flare observations.

- We have argued that recent high spatial observations at HXRs, specially those by provide strong evidence for the presence of turbulence in the acceleration sites of flares in the corona near the top of the flaring loops.

- It appears that this turbulence can be the main agent of acceleration in flares with a resultant electron spectra that agree with many of the recent observations.

- We have shown that the SA by turbulence can account for high relative efficiency of acceleration of electrons vs protons in solar flares that agrees with their relative numbers as deduced by the HXR emission by electrons and gamma-ray emission by protons.

- Similarly, we have shown that this model can account quantitatively for the long standing puzzle of extreme enhancement of ${ }^{3} \mathrm{He}$ abundances in the low fluence impulsive SEP events relative to that of ${ }^{4} \mathrm{He}$ and reproduce their observed spectra accurately.

- However, there are some aspects of SEPs that the SA model cannot account for. One is the fact that the ratio of electron to proton energy ratios in SEPs is smaller than in flares. Another is that ${ }^{4} \mathrm{He}$ spectra resulting in from SA by turbulence in the flare region for high fluence-gradual events are too soft compared with observations. We have proposed that the hybrid model described above can resolve these discrepancies, whereby the flare accelerated particles are re-accelerated by CME shocks which are more likely to be present in bigger flares.

Acknowledgment: I thank colleagues Siming Liu and Wei Liu for production of some of the results presented here and graduate student Qingrong Chen for help in preparation 
of this manuscript. The research providing the result presented here were supported by previous NSF and NASA grants and current NASA grant NNX10AC06G.

\section{REFERENCES}

Ackermann, M., Ajello, M., Allafort, A., et al. 2012, ApJ, 745, 144

Achterberg, A. 1979, A\&A, 76, 276

Axford, W. I., Leer, E., \& Skadron, G. 1978, Cosmophysics, 125

Bell, A. R. 1978, MNRAS, 182, 147

Beresnyak, A., Jones, T. W., \& Lazarian, A. 2009, ApJ, 707, 1541

Blandford, R., \& Eichler, D. 1987, Phys. Rep., 154, 1

Blandford, R. D., \& Ostriker, J. P. 1978, ApJ, 221, L29

Brunetti, G., \& Lazarian, A. 2007, MNRAS, 378, 245

Cassak, P. A., Drake, J. F., \& Shay, M. A. 2006, ApJ, 644, L145

Cowsik, R.\& Sarkar, S. 1984, MNRAS, 207, 745

Cliver, E. W., \& Ling, A. G. 2007, ApJ, 658, 1349

de Gouveia dal Pino, E. M., \& Lazarian, A. 2005, A\&A, 441, 845

Desai, M. I., Mason, G. M., Dwyer, J. R., et al. 2003, ApJ, 588, 1149

Diamond, P. H., \& Malkov, M. A. 2007, ApJ, 654, 252

Drake, J. F., Swisdak, M., Che, H., \& Shay, M. A. 2006, Nature, 443, 553

Drury, L. 1983, Space Sci. Rev., 36, 57

Dung, R., \& Petrosian, V. 1994, ApJ, 421, 550

Eilek, J. A. 1979, ApJ, 230, 373

Fan, Z., Liu, S., Fryer, C. L. 2009, MNRAS, 406, 1337

Fermi, E. 1949, Physical Review, 75, 1169

Fermi, E. 1954, ApJ, 119, 1

Giacalone, J. 2005a, ApJ, 624, 765

Giacalone, J. 2005b, ApJ, 628, L37

Hall D. E, \& Sturrock P. A. 1967, Plasma Phys., 10, 2620

Hamilton, R. J., \& Petrosian, V. 1992, ApJ, 398, 350 
Ho, G. C., Roelof, E. C., \& Mason, G. M. 2005, ApJ, 621, L141

Holman, G. D. 1985, ApJ, 293, 584

Jiang, Y. W., Liu, S., Liu, W., \& Petrosian, V. 2006, ApJ, 638, 1140

Jones, F. C. 1994, ApJS, 90, 561

Jones, F. C., \& Ellison, D. C. 1991, Space Sci. Rev., 58, 259

Kirk, J. G., Schneider, P., \& Schlickeiser, R. 1988, ApJ, 328, 269

Krucker, S., \& Lin, R. P. 2008, ApJ, 673, 1181

Krymskii, G. F. 1977, Akademiia Nauk SSSR Doklady, 234, 1306

Krymsky, G. F., Kunming, A. I., Petulant, S.I. \& Turnover, A.A., 1979, Proc. 16th Int. Conf. on Cosmic Rays (Kyoto) 2, 39

Kulsrud, R. M., \& Ferrari, A. 1971, Ap\&SS, 12, 302

Lagage, P. O., \& Cesarsky, C. J. 1983, A\&A, 125, 249

Lazarian, A., \& Vishniac, E. T. 1999, ApJ, 517, 700

Lazarian, A., Petrosian, V., Yan, H., \& Cho, J. 2003, arXiv:astro-ph/0301181

Lacombe, C. 1977, A\&A, 54, 1

Lee, M. A. 2005, ApJS158, 38

Li, H., \& Miller, J. A. 1997, ApJ, 478, L67

Liu, S., Fan, Z.-H., Fryer, C. L., Wang, J.-M., \& Li, H. 2008, ApJ, 683, L163

Liu, S., Melia, F., \& Petrosian, V. 2006, ApJ, 636, 798

Liu, S., Petrosian, V., \& Mason, G. M. 2004, ApJ, 613, L81

Liu, S., Petrosian, V., \& Mason, G. M. 2006, ApJ, 636, 462

Liu, S., Petrosian, V.\& Melia, F. 2006, ApJ, 611, 101L

Liu, S., Petrosian, V., Melia, F., \& Fryer, C. L. 2006, ApJ, 648, 1020

Liu, S., Qian, L., Wu, X.-B., Fryer, C. L., \& Li, H. 2007, ApJ, 668, L127

Liu, W., Jiang, Y. W., Liu, S., \& Petrosian, V. 2004, ApJ, 611, L53

Liu, W., Jiang, Y. W., Petrosian, V., \& Metcalf, T. R. 2003, Bulletin of the American Astronomical Society, 35, 839

Liu, W., Petrosian, V., Dennis, B. R., \& Jiang, Y. W. 2008, ApJ, 676, 704

Litvinenko, Y. E. 1996, ApJ, 462, 997

Litvinenko, Y. E. 2003, Sol. Phys., 212, 379 
Malkov, M. A., \& O'C Drury, L. 2001, Reports on Progress in Physics, 64, 429

Mason, G. M., Dwyer, J. R., \& Mazur, J. E. 2000, ApJ, 545, L157

Mason, G. M., Mazur, J. E., \& Dwyer, J. R. 2002, ApJ, 565, L51

Mason, G. M., Reames, D. V., von Rosenvinge, T. T., Klecker, B., \& Hovestadt, D. 1986, ApJ, 303, 849

Masuda, S., Kosugi, T., Hara, H., Tsuneta, S., \& Ogawara, Y. 1994, Nature, 371, 495

Mazur, J. E., Mason, G. M., \& Klecker, B. 1995, ApJ, 448, L53

McTiernan, J. M., Kane, S. R., Loran, J. M., et al. 1993, ApJ, 416, L91

Melrose, D. B. 2009, arXiv:0902.1803

Miller, J. A. 2002, Multi-Wavelength Observations of Coronal Structure and Dynamics, 387

Miller, J. A., \& Reames, D. V. 1996, American Institute of Physics Conference Series, 374,450

Miller, J. A., \& Roberts, D. A. 1995, ApJ, 452, 912

Murphy, R. J., Dermer, C. D., \& Ramaty, R. 1987, ApJS, 63, 721

Ng, C. K., \& Reames, D. V. 1994, ApJ, 424, 1032

Park, B. T., Petrosian, V., \& Schwartz, R. A. 1997, ApJ, 489, 358

Pryadko, J. M., \& Petrosian, V. 1999, ApJ, 515, 873 (PP97)

Petrosian, V. 2008, arXiv:0808.1757

Petrosian, V. 2001, ApJ, 557, 560

Petrosian, V. \& Chen, Q. 2010, ApJ712, 131

Petrosian, V., \& Donaghy, T. Q. 1999, ApJ, 527, 945

Petrosian, V., Donaghy, T. Q., \& McTiernan, J. M. 2002, ApJ, 569, 459

Petrosian, V., \& East, W. E. 2008, ApJ, 682, 175

Petrosian, V., Jiang, Y. W., Liu, S., Ho, G. C., \& Mason, G. M. 2009, ApJ, 701, 1

Petrosian, V., \& Liu, S. 2004, ApJ, 610, 550

Piana, M., et al. 2003, ApJ Letters, 595, 127

Piana, M., et al. 2007, ApJ, 665, 846

Pryadko, J. M., \& Petrosian, V. 1997, ApJ, 482, 774

Ramaty, R. 1979, Particle Acceleration Mechanisms in Astrophysics, 56, 135 
Reames, D. V., Barbier, L. M., von Rosenvinge, T. T., et al. 1997, ApJ, 483, 515

Reames, D. V., Meyer, J. P., \& von Rosenvinge, T. T. 1994, ApJS, 90, 649

Scott, J. S. \& Chevalier, R. A. 1975, ApJ, 197, L5

Shih, A. Y., Lin, R. P., \& Smith, D. M. 2009, ApJ, 698, L152

Sironi, L., \& Spitkovsky, A. 2009, ApJ, 707, L92

Speiser, T. W. 1970, Planet. Space Sci., 18, 613

Spitzer, L. 1962, Physics of Fully Ionized Gases, New York: Interscience (2nd edition), 1962 ,

Stawartz, L. \& Petrosian, V. 2008 ApJ681, 1725

Sturrock, P. A. 1966, Physical Review, 141, 186

Sui, L., \& Holman, G. D. 2003, ApJ, 596, L251

Tsuneta, S. 1985, ApJ, 290, 353

Tylka, A. J., \& Lee, M. A. 2006, ApJ, 646, 1319

Virtanen, J. J. P., \& Vainio, R. 2005, ApJ, 621, 313

Yan, H., \& Lazarian, A. 2002, Physical Review Letters, 89, 1102

Zenitani, S., \& Hoshino, M. 2005, ApJ, 618, L111

This preprint was prepared with the AAS IATEX macros v5.2. 